\title{
A Fast Algorithm for Monostatic RCS based on LU Decomposition
}

\author{
Xie Xiaogang, Yu Tongbin, Qian Zuping and \\ Zhang Lei \\ Institute of Communications Engineering, PLAUST \\ Nanjing, China, 210007 \\ E-mail: xie_xiaogang@163.com
}

\author{
Liu Ying \\ Xi'an Communications Institute \\ Xi'an, China, 710106 \\ E-mail: danfer_ice@163.com
}

\begin{abstract}
Compared with bistatic RCS, the monostatic RCS calculation is more difficult for the needs of surface current on scatterer induced by the incident wave of every single angle, so the calculation burthen is very large. Based on the character of "impedance matrix of MOM is invariable whether the angle of incident wave was changed", this paper proposes a fast algorithm for monostatic RCS based on LU decomposition. Numerical results show out that this algorithm can compress the computation burthen of monostatic RCS significantly, and this algorithm is easy to operate.
\end{abstract}

Keywords-MOM, RWG basis, monostatic RCS, LU decomposition

\section{INTRODUCTION}

The calculation for monostatic is complicated than bistatic RCS because of the needs of surface current on scatterer or equivalent field on Huygens extrapolated boundary for each incident angle. If the scatterer was complicated, the calculation burthen will be very large. So the studies almost focus on bistatic RCS more than monostatic one ${ }^{[1-2]}$. In recent years, some scholars have made some progress in monostatic RCS calculation ${ }^{[3-6]}$, but there is some space to ideally in the region of accuracy or computation burthen.

In the beginning of 1980's, Rao and other scholars proposed the famous Rao-Wilton-Glisson basis (RWG-basis) function $^{[7]}$. The RWG-basis caught lots of attention for its flexibility and practicability since it was proposed, and has been applied successfully in many fields ${ }^{[8-10]}$. The impedance matrix of MOM based on RWG-basis has a characteristic that the impedance matrix is invariable whether the incident angle changes. Based on this specialty, references [11] proposed a monostatic RCS calculation method based on Gauss Elimination method. This method simplified the computation process of monostatic RCS but not easy to implement. This paper proposes an algorithm based on LU decomposition for monostatic RCS calculation. This new algorithm transforms the original equation into triangle matrix equation by LU decomposition of the impedance matrix, and it cut down the computation burthen of the matrix equation significantly. Simulation results show out that this algorithm can shorten the computation time significantly.

\section{MOMENT EQUATIONS BASED ON RWG-BASIS}

Based on the boundary conditions of tangential electric field continuality on metal surface, so there will be $\mathrm{n}^{\hat{n} \times \bar{E}(\bar{r})=0}$, which is $\hat{n} \times\left(E^{i}+E^{s}\right)=0$. Where $E^{i}$ is the incident plane wave, the scattering field induced by $E^{i}$ can be express as $E^{s}=-j \omega A-\nabla \Phi$, so the fields integral equation can be express as:

$$
-E_{\mathrm{tan}}^{i}=(-j \omega A-\nabla \Phi)_{\tan }
$$

where $A$ and $\Phi$ are the magnetic vector potential function and electric scalar potential function respectively.

Formula (1) can be solved by moment method and the induced surface current $\mathrm{J}$ can be expressed as ${ }^{J=\sum_{n=1}^{N} I_{n} f_{n}(r)}$, where $I_{n}$ is current coefficient to be solved, and it is corresponding to the normal current density of the common edge, $f_{n}$ is RWG-basis, and $N$ is the amount of common edges.

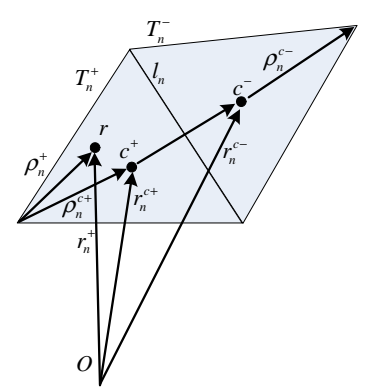

Fig 1 RWG-basis Function

RWG-basis is sub domain base function, and each basis function is associated with an interior edge of the triangle patches pair and it is zero everywhere on the surface of scatterer, except in the two triangles $T_{n}^{ \pm}$attached to that interior edge $^{l_{n}}$. Fig1 shows two such triangles, $T_{n}^{+}$and ${ }^{T_{n}^{-}}$, corresponding to the $n$th edge of a triangulated surface modeling. The basis function $f_{n}$ is used to approximately represent the surface current. In reference [7], the vector basis function associated with the $n$th edge was defined as 


$$
f_{n}(r)=\left\{\begin{array}{cc}
\frac{l_{n}}{2 A_{n}^{+}} \rho_{n}^{+}, & r \in T_{n}^{+} \\
\frac{l_{n}}{2 A_{n}^{-}} \rho_{n}^{-}, & r \in T_{n}^{-} \\
0, & \text { others }
\end{array}\right.
$$

In formula (2), ${ }_{n}$ is the length of the edge and $A_{n}^{ \pm}$is the area of triangle ${ }^{T_{n}^{ \pm}}$.The plus or minus designation of the triangles is determined by the choice of a positive current reference direction for the $n$th edge, the reference for which is assumed to be $T_{n}^{+} \rightarrow T_{n}^{-}, \rho_{n}^{+}$is the vector on $T_{n}^{+}$which is from vertex of ${ }^{l_{n}}$ to any points in ${ }^{T_{n}^{+}}, \rho_{n}^{-}$is the vector on $T_{n}^{-}$which is from vertex of $l_{n}$ to any points in $T_{n}^{-}, r$ is the vector form origin to any points. The function basis $f_{n}$ out of $T_{n}^{ \pm}$are all 0.

Using Galerkin's method to take formula (1) and $f_{m}$ for inner product, we can get

$$
\left(E^{i}, f_{m}\right)=j \omega\left(A, f_{m}\right)+\left(\nabla \Phi, f_{m}\right)
$$

and then arrange formula (3) as $\mathbf{Z I}=\mathbf{V}$ form as

$$
\begin{gathered}
z_{m n}=l_{m}\left[j \omega\left(A_{m n}^{+} \cdot \frac{\rho_{m}^{c+}}{2}+A_{m n}^{-} \cdot \frac{\rho_{m}^{c-}}{2}\right)+\Phi_{m n}^{-}-\Phi_{m n}^{+}\right] \\
v_{m}=l_{m}\left[E_{m}^{c+} \cdot \frac{\rho_{m}^{c+}}{2}+E_{m}^{c-} \cdot \frac{\rho_{m}^{c-}}{2}\right]
\end{gathered}
$$

where

$$
\begin{gathered}
A_{m n}^{ \pm}=\frac{\mu}{4 \pi} \int_{s} f_{n}\left(r^{\prime}\right) \frac{e^{-j k R_{m}^{ \pm}}}{R_{m}^{ \pm}} d s^{\prime} \\
\Phi_{m n}^{ \pm}=-\frac{1}{4 \pi j \omega \varepsilon} \int_{s} \nabla_{s} \cdot f_{n}\left(r^{\prime}\right) \frac{e^{-j k k_{m}^{ \pm}}}{R_{m}^{ \pm}} d s^{\prime} \\
R_{m}^{ \pm}=\left|r_{m}^{c \pm}-r^{\prime}\right| \\
E_{m}^{ \pm}=E^{i}\left(r_{m}^{c \pm}\right)
\end{gathered}
$$

(4) lower triangular matrix, $\mathbf{U}=\left\{u_{i j}\right\}$ is upper triangular matrix, then the corresponding elements in $\mathbf{L}, \mathbf{U}$ can be expressed as formula (10) and (11), where, element $u_{k j}$ in row $k$ in $\mathbf{U}_{\text {can }}$ be expressed as:

$$
u_{k j}=a_{k j}-\sum_{r=1}^{k-1} l_{k r} u_{r j}, \quad j=k, k+1, \ldots, n
$$

(6) where $^{k}=1,2, \ldots, n$; element ${ }^{l_{i k}}$ in column $k$ in $\mathbf{L}$ can be expressed as:

$$
l_{i k}=\frac{a_{i k}-\sum_{r=1}^{k-1} l_{i r} u_{r k}}{u_{k k}}, \quad i=k+1, \ldots, n
$$

where $i=1,2, \ldots, n$, and $\sum_{r=1}^{0}=0$

Using formula (10) and (11) alternatively and successively, we can solve all the elements in $\mathbf{L}$ and $\mathbf{U}$, and all these elements can be saved in the corresponding position in matrix A. This process can be programmed easily, and during the computation process, the elements in matrix $\mathbf{A}_{\text {can be replaced by elements in matrix }} \mathbf{L}, \mathbf{U}$ successively. And it is no need to use new matrix to save $\mathbf{L}, \mathbf{U}$, so the storage is more efficient. 
Based on the LU decomposition in the former step, $\mathbf{L y}=\mathbf{b}$ and $\mathbf{U x}=\mathbf{y}$ can be solved by successive substitution method, the computation method can be expressed as follow:

$$
\begin{gathered}
y_{i}=b_{i}-\sum_{r=1}^{i-1} l_{i r} y_{r}, \quad i=1,2, \ldots, n \\
x_{i}=\frac{y_{i}-\sum_{r=i+1}^{n} u_{i r} x_{r}}{u_{i i}}, \quad i=n, n-1, \ldots, 1
\end{gathered}
$$

The original equation can be solved by formula (12) and (13). For solving RWG moment equations, the impedance matrix should be transformed from $\mathbf{Z I}=\mathbf{V}_{1}$ to $\mathbf{Z I}=\mathbf{V}_{2}$ if the angle of incident wave changed, where $\mathbf{V}^{(2)}=\left(v_{1}^{(2)}, \ldots, v_{n}^{(2)}\right)^{T}$. In these two equations, that the matrix $\mathbf{Z}$ on the left side is invariable and $\mathbf{V}$ on the right side changes only. So we can using $\mathbf{L}$ and $\mathbf{U}$, which are known already, to solve the new equation for simplify the computation procedure of monostatic RCS.

\section{NUMERICAL RESULTS}

\section{A. Monostatic RCS of Rectangle Metal Plate}

Fig2 shows the monostatic RCS of rectangle metal plate, which is located in XOY plane, and calculated by different algorithm. Where the incident angle represents the angle between incident wave vector $\hat{k}$ and $\mathbf{X}$ axis, and it is changed in XOY plane; Electric field $E_{i}$ is parallel to XOY plane and its amplitude is 1; Frequency of incident wave is $300 \mathrm{MHz}$ and the size of the metal plate is $1 \mathrm{~m} \times 1 \mathrm{~m}$. Simulation results show out that the RCS results of Gauss Elimination method and LU decomposition method consistent with each other, which proves the correctness of this new algorithm.

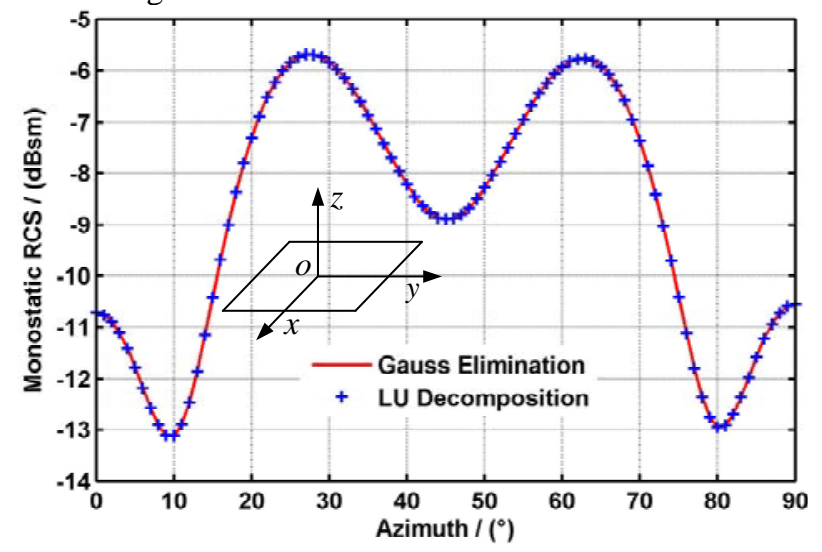

Fig2 monostatic RCS of Rectangle metal plate

\section{B. Monostatic RCS of Metal Cubic}

Fig3 shows the monostatic RCS of metal cubic in different algorithm. Where the incident angle represents the angle between incident wave vector $\hat{k}$ and $\mathrm{Z}$ axis, and it is changed in YOZ plane; The direction of Electric field $E_{i}$ is $\hat{X}$ and its amplitude is 1; Frequency of incident wave is $300 \mathrm{MHz}$ and the size of metal cubic is $1 \mathrm{~m} \times 1 \mathrm{~m} \times 1 \mathrm{~m}$. Simulation results show out that RCS computed by Gauss Elimination method and LU decomposition method consistent with each other very well, and they both consistent with the result implemented by Ansoft HFSS.

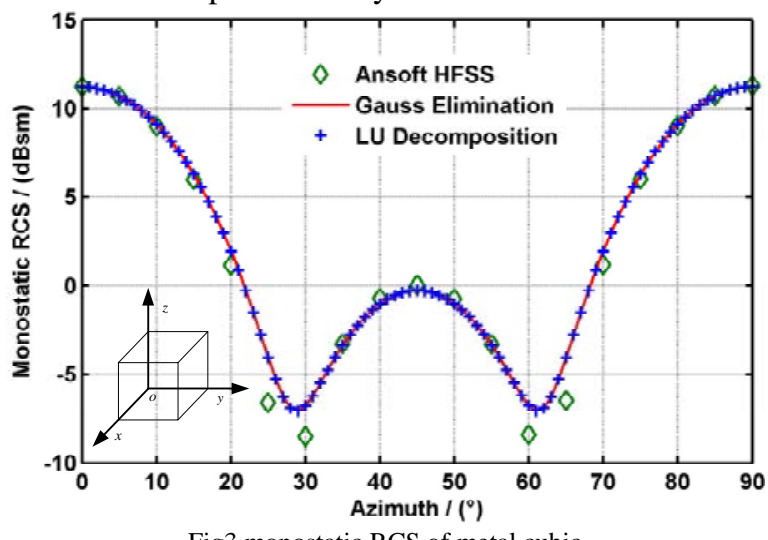

Fig3 monostatic RCS of metal cubic

\section{Analysis of Computation Burthen}

If solving the equation completely by LU decomposition, the computation burthen is equivalent to Gauss Elimination method $^{[13]}$, so there is no advantage by adopting this method. But for monostatic RCS, the procedure of LU decomposition for impedance matrix is carried on only once and this will compress the computation burthen remarkably.

Fig4 shows the computation times of Gauss Elimination method and LU decomposition method expended in former simulation of metal cubic respectively. We can draw a conclusion that compared to traditional Gauss Elimination method, the proposed algorithm in this paper can compress the computation time to almost $10 \%$ of the Gauss Elimination method.

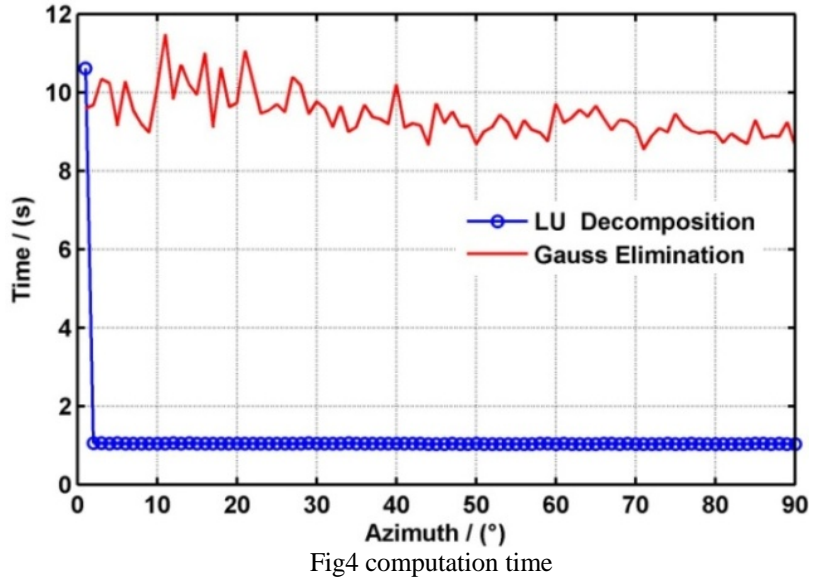




\section{CONCLUSION}

Based on the character of "impedance matrix of MOM is invariable whether the angle of incident wave was changed", this paper proposes a fast algorithm for monostatic RCS based on LU decomposition. The proposed algorithm predigest a complex matrix equation into two triangle matrix equations by LU decomposition, and predigest the computation procedure of monostatic RCS in further by using the invariable characteristic of the impedance matrix. Numerical results show out that the proposed algorithm can compress the computation burthen of monostatic RCS significantly, and it is very easy to operate.

\section{ACKNOWLEDGMENT}

This work was supported by the National Natural Science Foundation of China under grant (No. 61271103).

\section{REFERENCES}

[1] Fangzhi Geng, Dongyu Xia, Huan He, Yingfeng Pan, Chunzhu Dong, "RCS Analysis Considering Multi-Reflection Using SBR/RDN Method Based on RWG Basis Function”. ICMMT2008

[2] JunhoY eo, Sinan Koksoy, V. V. S. Prakash, and Raj Mittra. "Efficient Generation of Method of Moments Matrices Using the Characteristic Function Method”. IEEE Trans. Ant. \&Prop. , VOL. AP-52, NO. 12, pp.3405-3410,DEC 2004

[3] Wan Jixiang, Zhang Yu, Xiang Tieming, et a1. Rapid solutions of monostatic RCS using FMM with adaptive MBPE technique. Chinese Journal of Radio Science, 2004, 19(1) : 7276 .
[4] Wang Haibin. An efficient scheme for calculating high frequency EM scatterering from complex objects[J]. Chinese Journal of Radio Science, 2009, 24(1) : 65-70.

[5] Xu Lijun, Lin Baoqin, Yuan Naichang. Application of AWE to fast calculation of wide angle RCS of 2-D conducting cylinder covered by dielectric. Journal of Microwaves, 2004, 20(3) : 6-9.

[6] Tan Yunhua, Zhou Lezhu. Using the fast multipole method to analyze monostatic RCS of electrically large 3-D multi-conducting bodies . Journal of Beijing University of Posts and Telecommunications, 2004, 40(5):823-829.

[7] RAO S M. WILTON D R, GLISSON A W, "Electromagnetic scatterering by surfaces of arbitrary shape,” IEEE Trans. Ant. \&Prop., vol.AP-30, no.3, pp.409-418, MAY 1982.

[8] Abdulkadir C Y, A. Arif Ergin, "Exact Evaluation of Retarded-Time Potential Integrals for the RWG Bases," IEEE Trans. Ant. \&Prop. vol.AP-54, no.5, pp. 1496-1502, MAY 2006.

[9] Pasi Y-O and Matti Taskinen, "Calculation of CFIE Impedance Matrix Elements With RWG and $\times$ RWG Functions," IEEE Trans. Ant. \&Prop. , vol.AP-51, no.8, pp.1837-1846, Aug. 2003

[10] Xuanx Hui Wu, Ahmed A. Kishk, and Allen W. Glisson, “An RWG Basis Function Based Near- to Far-Field Transformation for 2-D Triangular-Grid Finite Difference Time Domain Method,” IEEE Trans. Ant. \&Prop., vol.AP-54, no.6, pp.1901-1905, Jun. 2006.

[11] XU Xiao-fei, CAO Xiang-yu, GAO Jun, WU Jun-hui. "Fast Calculation Method of Target' S Monostatic RCS". Journal of Beijing University of Posts and Telecommunications, Vol.34 No.5 Oct. 2011

[12] Zhang xian-da, "Matrix analysis and applications". Beijing: TSINGHUA University Press. Sep. 2004

[13] Li Qing-yang, Guan Zhi, Bai Feng-shan. "Principle of Numerical Calculation”. Beijing: TSINGHUA University Press. Sep. 2000 